\title{
Hypergeometric Equations and Weighted Projective Spaces
}

\author{
Alessio Corti \\ Department of Mathematics, Imperial College London \\ Huxley Building, 180 Queen's Gate \\ London SW7 2AZ, UK \\ Vasily Golyshev \\ Number Theory Section, Steklov Mathematical Institute \\ Gubkina str. 8, \\ 119991, Moscow, Russia
}

$30^{\text {th }}$ June, 2006

\begin{abstract}
We compute the Hodge numbers of the polarised (pure) variation of Hodge structure $\mathbb{V}=\operatorname{gr}_{n-1}^{W} R^{n-1} f ! \mathbb{Z}$ of the Landau-Ginzburg model $f: Y \rightarrow \mathbb{C}$ mirror-dual to a weighted projective space $w \mathbb{P}^{n}$ in terms of a variant of Reid's age function of the anticanonical cone over $w \mathbb{P}^{n}$. This implies, for instance, that $w \mathbb{P}^{n}$ has canonical singularities if and only if $h^{n-1,0} \mathbb{V}=1$. We state a conjectural formula for the Hodge numbers of general hypergeometric variations.

We show that a general fibre of the Landau-Ginzburg model is birational to a Calabi-Yau variety if and only if a general anticanonical section of $w \mathbb{P}$ is Calabi-Yau. We analyse the 104 weighted 3-spaces with canonical singularities, and show that a general anticanonical section is not a K3 surface exactly in those 9 cases where a generic fibre of the Landau-Ginzburg model is an elliptic surface of Kodaira dimension 1.
\end{abstract}




\section{Contents}

1 Introduction and results. 2

1.1 Some hvpergeometric local svstems. . . . . . . . . . 2

1.2 Hodge numbers of hvpergeometric local svstems . . . . 4

1.3 Relation to the Reid-Tai criterion . . . . . . . . . 5 5

1.4 Relation to the McKav correspondence . . . . . . . . 6

1.5 Mirror svmmetrv . . . . . . . . . . . . . 7

1.6 Calabi-Yau weighted hvpersurfaces . . . . . . . . . 7

2 Proof of Theorems 1.1 and $1.3 \quad 10$

3 Proof of Theorems 1.13, 1.15, 1.17 12

3.1 Toric varieties . . . . . . . . . . . . . . . 12

3.2 Discrepancies and canonical singularities . . . . . . . . 14

3.3 Proof of Theorem $1.15 \ldots \ldots \ldots \ldots$

3.4 Proof of Theorem 1.13 . . . . . . . . . . . . 18

3.5 Proof of Theorem $1.17 \ldots \ldots \ldots \ldots$

$\begin{array}{ll}\text { References } & 22\end{array}$

\section{Introduction and results.}

\subsection{Some hypergeometric local systems.}

Fix positive integers (called weights) $w_{0}, \ldots, w_{n}$ and write $d=\sum w_{i}$. We assume that $\operatorname{hcf}\left(w_{0}, \ldots, \widehat{w_{i}}, \ldots, w_{n}\right)=1$ for all $i$, that is, the set of weights is well-formed. The main object of interest in this paper is the regular hypergeometric operator on $\mathbb{C}^{\times}$:

$$
\begin{aligned}
H=\prod_{i=0}^{n} w_{i}^{w_{i}} D\left(D-\frac{1}{w_{i}}\right) \cdots & \left(D-\frac{w_{i}-1}{w_{i}}\right)- \\
& \quad-t d^{d} D\left(D+\frac{1}{d}\right) \cdots\left(D+\frac{d-1}{d}\right)
\end{aligned}
$$

where $D=t d / d t$. Note that the symbol of $H$ is $\prod w_{i}^{w_{i}}-t d^{d}$; therefore, $H$ is singular at $t=\lambda=\left(\prod w_{i}^{w_{i}}\right) /\left(d^{d}\right)$. More precisely, we are interested in the operator $H^{\text {red }}$ obtained removing from each of the two summands on the right hand side of Equation (11) one copy of every common factor; it is known that $H^{\text {red }}$ is irreducible. 
In Theorem 1.1 we construct a precise Picard-Fuchs interpretation of the local system of solutions of the ordinary differential equation $H^{\text {red }} \varphi=0$ and, in Theorem 1.3, we compute its Hodge numbers.

Consider the variety

$$
Y=\left\{\begin{array}{l}
\prod_{i=0}^{n} y_{i}^{w_{i}}=t \\
\sum_{i=0} y_{i}=1
\end{array} \quad \subset \quad \mathbb{C}^{\times n+1} \times \mathbb{C}^{\times}\right.
$$

(where $y_{0}, \ldots, y_{n}$ and $t$ are coordinates on $\mathbb{C}^{\times n+1}$ and $\mathbb{C}^{\times}$) and the pencil $f=\operatorname{pr}_{2}: Y \rightarrow \mathbb{C}^{\times}$.

Theorem 1.1. Denote by $\mathbb{V}_{\mathbb{R}}$ the real local system of solutions of the ordinary differential equation $H^{\text {red }} \varphi=0^{1}$; then

$$
\mathbb{V}_{\mathbb{R}}=\operatorname{gr}_{n-1}^{W} R^{n-1} f_{!} \mathbb{R}_{Y}
$$

In particular, this naturally gives $\mathbb{V}_{\mathbb{R}}$ the structure of an integer local system $\mathbb{V}_{\mathbb{Z}}$.

Remark 1.2. Katz constructs a pure $\ell$-adic realisation of the local system $\mathbb{V}_{\mathbb{R}}\left[\right.$ Kat90, Chapter 8]; let us denote it by $\mathbb{V}_{\mathbb{Q}_{\ell}}$; our proof of Theorem 1.1 also shows that $\mathbb{V}_{\mathbb{Q}_{\ell}}=\operatorname{gr}_{n-1}^{W} R^{n-1} f_{!} \mathbb{Q}_{\ell Y}$.

By Theorem 1.1, $\mathbb{V}_{\mathbb{Z}}$ supports a polarised variation of (integer) Hodge structure. Motivated by mirror symmetry, in Theorem [1.3 we calculate the Hodge numbers of this PVHS in terms of the geometry of the anticanonical affine cone

$$
A=\operatorname{Spec} \oplus_{m \geq 0} H^{0}(w \mathbb{P},-m K)
$$

over the weighted projective space $w \mathbb{P}=\mathbb{P}\left(w_{0}, \ldots, w_{n}\right)$. Concretely, set $N=\mathbb{Z}\left(w_{0} / d, \ldots, w_{n} / d\right)+\mathbb{Z}^{n+1}$ and let $M=\operatorname{Hom}(N, \mathbb{Z})$; with this notation, $A$ is the affine toric variety $\operatorname{Spec} \mathbb{C}\left[M \cap I^{*}\right]$ attached to the positive orthant $I=\mathbb{R}_{+}^{n+1} \subset N_{\mathbb{R}}$; equivalently, $A=\mathbb{C}^{n+1} / \mu_{d}$ is the quotient of $\mathbb{C}^{n+1}$ by $\mu_{d}$ acting diagonally with weights $w_{0}, \ldots, w_{n}$.

We fix the linear form

$$
l: N \rightarrow \mathbb{Z} \quad \text { defined as } l\left(\alpha_{0}, \ldots, \alpha_{n}\right)=\sum \alpha_{i} .
$$

If $a$ is an integer, we denote by $\bar{a}$ the smallest positive integer mod $d$, that is, $0 \leq \bar{a}<d$ and $\bar{a} \equiv a \bmod d$. Every element

$$
\left(\frac{a_{0}}{d}, \ldots, \frac{a_{n}}{d}\right) \in N \text { has a unique representative }\left(\frac{\overline{a_{0}}}{d}, \ldots, \frac{\overline{a_{n}}}{d}\right)
$$

\footnotetext{
${ }^{1}$ It is well-known from [BH89] that the (complex) local system of solutions of $H^{\text {red }} \varphi=0$ has a natural real structure.
} 
$\bmod \mathbb{Z}^{n+1}$ in the unit box $[0,1)^{n+1} \subset \mathbb{R}^{n+1}$; in particular, this identifies the cyclic group $\mathbb{Z} / d=N / \mathbb{Z}^{n+1}$ generated by $\left(w_{0} / d, \ldots, w_{n} / d\right)$ with $N \cap[0,1)^{n+1}$. We denote by $a: N / \mathbb{Z}^{n+1} \rightarrow[0, n] \cap \mathbb{Z}$ the age function of Reid:

$$
a\left(\frac{a_{0}}{d}, \ldots, \frac{a_{n}}{d}\right)=l\left(\frac{\overline{a_{0}}}{d}, \ldots, \frac{\overline{a_{n}}}{d}\right)=\frac{1}{d} \sum_{i=0}^{n} \overline{a_{i}} .
$$

We denote by $\left(N / \mathbb{Z}^{n+1}\right)^{0}=(\mathbb{Z} / d)^{0}$ the set of classes not contained in a coordinate hyperplane:

$$
\begin{aligned}
(\mathbb{Z} / d)^{0}=\{0 \leq & \left.k<d \mid d \text { divides no } k w_{i}\right\}= \\
= & \left\{\left(\frac{\overline{k w_{0}}}{d}, \ldots, \frac{\overline{k w_{n}}}{d}\right) \mid \text { no } \overline{k w_{i}}=0\right\}=\left(N / \mathbb{Z}^{n+1}\right)^{0},
\end{aligned}
$$

and, for $j=1, \ldots, n$, we denote by

$$
a_{j}^{s}=\operatorname{card}(\mathbb{Z} / d)^{0} \cap a^{-1}(j)
$$

the number of elements of strict age $j$; alternatively, $a_{j}^{s}$ is the number of elements $\mathbf{v} \in N \cap(0,1)^{n+1}$ with $l(\mathbf{v})=j$. Our main result is:

Theorem 1.3. For $j=1, \ldots, n$ :

$$
\operatorname{rk} \mathcal{H}^{n-j, j-1}\left(V_{\mathbb{Z}} \otimes \mathcal{O}_{\mathbb{C}^{\times} \backslash\{\lambda\}}\right)=h^{n-j, j-1}\left(H_{c}^{n-1} Y_{t}\right)=a_{j}^{s}
$$

We prove Theorems 1.1 and 1.3 in $\$ 2$ In the remaining part of the Introduction, after a brief discussion of some conjectures and corollaries in the light of mirror symmetry, we state our next results Theorems 1.131 .15 and 1.17

\subsection{Hodge numbers of hypergeometric local systems}

We expect that we will soon be able to announce progress on the following natural conjecture:

Conjecture 1.4. For real numbers $0 \leq \alpha_{0} \leq \cdots \leq \alpha_{n-1}<1$ and $0 \leq \beta_{0} \leq \cdots \leq \beta_{n-1}<1$ such that $\alpha_{j} \neq \beta_{k}$ (all $j, k$ ) and the two sets $\left\{\exp 2 \pi i \alpha_{j}\right\},\left\{\exp 2 \pi i \beta_{k}\right\}$ are stable under complex conjugation, consider the irreducible regular hypergeometric differential operator:

$$
H\left(\left\{\alpha_{j}\right\} ;\left\{\beta_{k}\right\}\right)=\prod\left(D-\alpha_{j}\right)-t \prod\left(D-\beta_{k}\right),
$$


write

$$
p(k)=\operatorname{card}\left\{j \mid \alpha_{j}<\beta_{k}\right\}-k,
$$

and set $p_{+}=\max \{p(k)\}, p_{-}=\min \{p(k)\}$. The local system of solutions of the ordinary differential equation $H \varphi=0$ supports a real polarised variation of Hodge structure of weight $p_{+}-p_{-}$and Hodge numbers

$$
h^{j-p_{-},-j+p_{+}}=\operatorname{card} p^{-1}(j) .
$$

The main evidence for the conjecture is:

Proposition 1.5. Given weights $w_{0}, \ldots, w_{n}$ and $d=\sum w_{i}$ as before, set

$$
A=\bigcup_{i=0}^{n}\left\{\frac{k}{w_{i}} \mid k=0, \ldots, w_{i}-1\right\}, \quad B=\left\{\frac{k}{d} \mid k=0, \ldots, d-1\right\},
$$

and $\left\{\alpha_{j}\right\}=A \backslash(A \cap B),\left\{\beta_{j}\right\}=B \backslash(A \cap B)$. Then the assertion of Conjecture 1.4 holds.

Proof. Assume $0<k / d<1$ is in $\left\{\beta_{j}\right\}$. This means precisely that $k \in(\mathbb{Z} / d)^{0}$. One has

$$
p(k)=k-\sum_{i}\left(\left[\frac{k w_{i}}{d}\right]+1\right), \quad \text { that is, } \quad p(k)=\left(\frac{\sum \overline{k w_{i}}}{d}\right)-n .
$$

In other words, $p(k)=a(k)-n$; the proposition now follows from Theorems 1.1 and 1.3.

Further evidence for the conjecture is given by the fact that it correctly predicts the signature of the polarisation of a hypergeometric local system computed by $\mathrm{BH} 89$. Theorem 4.5]. We are planning to cover the general case of the conjecture in our next publication.

\subsection{Relation to the Reid-Tai criterion}

Let us look at the case $k=1$ of Theorem 1.3. We view the affine hyperplane $N_{1}=N \cap l^{-1}(1)$ as a lattice by choosing the origin at

$$
\mathbf{e}=\left(\frac{w_{0}}{d}, \ldots, \frac{w_{n}}{d}\right) \text {. }
$$

We regard $N_{1}=\operatorname{Hom}\left(\mathbb{C}^{\times}, \mathbb{T}^{n}\right)$ as the lattice of one-parameter subgroups in a $n$-dimensional torus $\mathbb{T}^{n}$. The primitive vectors $\mathbf{e}_{0}, \ldots, \mathbf{e}_{n} \in$ 
$N_{1}$ generate a simplex $\Delta$ containing the origin $\mathbf{e}$, and the corresponding toric variety is the weighted projective space $w \mathbb{P}=\mathbb{P}\left(w_{0}, \ldots, w_{n}\right)$. The Reid-Tai criterion Rei87, §4] states that the nonzero integer points strictly inside $\Delta$ are in 1-to-1 correspondence with the geometric valuations $\nu$ of $w \mathbb{P}$ with discrepancy $a(\nu)<0$; therefore, we conclude:

\section{Corollary 1.6.}

$$
h_{c}^{n-1,0}\left(Y_{t}\right)=1+\operatorname{card}\{\nu \mid a(\nu)<0\} .
$$

In particular, $\mathbb{P}\left(w_{0}, \ldots, w_{n}\right)$ has canonical singularities if and only if $h_{c}^{n-1,0}\left(Y_{t}\right)=1$.

(We recall the notion of discrepancy and canonical singularities in Section 3.2 below.)

Remark 1.7. The linear function $l: N \rightarrow \mathbb{Z}$ of Equation (3) defines a grading on $\mathbb{C}[N]$ and a simplicial toric variety $\mathbb{P}=\operatorname{Proj} \mathbb{C}[N \cap I]$ where $I=\mathbb{R}_{+}^{n+1} \subset N_{\mathbb{R}}$ is the positive orthant; as we explain in the beginning of Section 3.5 the fibres $Y_{t}$ can naturally be seen to be hypersurfaces in the torus $\mathbb{T} \subset \mathbb{P}$; therefore, they are naturally compactified by simplicial hypersufaces $\overline{Y_{t}} \subset \mathbb{P}$. The construction makes it clear that a general $\overline{Y_{t}}$ is quasismooth; we conclude:

$$
h_{c}^{n-1,0}\left(Y_{t}\right)=h^{0}\left(\overline{Y_{t}}, K_{\overline{Y_{t}}}\right)=h^{0}\left(\widetilde{Y}_{t}, K_{\widetilde{Y}_{t}}\right)
$$

where $\widetilde{Y}_{t}$ is any nonsingular proper variety birational to $Y_{t}$.

\subsection{Relation to the McKay correspondence}

The anticanonical affine cone $A=\operatorname{Spec} \oplus_{n \geq 0} H^{0}(w \mathbb{P},-n K)$ over $w \mathbb{P}=$ $\mathbb{P}\left(w_{0}, \ldots, w_{n}\right)$ has, tautologically, Gorenstein singularities; it has cyclic quotient singularities; therefore, it has Gorenstein rational and hence canonical singularities. The McKay correspondence Rei02 states that, if $\widetilde{A} \rightarrow A$ is a crepant resolution, then:

$$
b^{2 j}(\widetilde{A})=\operatorname{rk} H^{2 j}(\widetilde{A}, \mathbb{Z})=a_{j}=\operatorname{card}(\mathbb{Z} / d) \cap a^{-1}(j) .
$$

More precisely, an element $g \in \mathbb{Z} / d$ of age $j$ determines a cohomology class in $H^{2 j}(\widetilde{A}, \mathbb{Z})$. We compare this statement with our Theorem 1.3 , stating that

$$
h^{n-j, j-1}\left(H_{c}^{n-1} Y_{t}\right)=a_{j}^{s}=\operatorname{card}(\mathbb{Z} / d)^{0} \cap a^{-1}(j) .
$$

The comparison suggests: 
Conjecture 1.8. $H \varphi=0$ is the quantum ordinary differential equation of the small quantum cohomology $Q H^{\bullet} \widetilde{A}$, and $H^{\text {red }} \varphi=0$ is a direct summand of it. Moreover, $Q H^{\bullet} \widetilde{A}$ is closely related to an appropriate variant of the small quantum orbifold cohomology $Q H_{\text {orb }}^{\bullet} A$.

See BGP for the discussion of a very special case. Note that $\widetilde{A}$ is non-compact and the cohomology ring $H^{\bullet}(\widetilde{A})$ does not satisfy Poincaré duality. Our Theorem 1.3 suggests that the subspace generated by the basis elements corresponding to elements in $(\mathbb{Z} / d)^{0}$ is a subring satisfying Poincaré duality: does this subring have a natural topological or geometric interpretation?

\subsection{Mirror symmetry}

We briefly discuss mirror symmetry for weighted projective spaces $w \mathbb{P}=\mathbb{P}\left(w_{0}, \ldots, w_{n}\right)$ in the context relevant to this paper. [CCLT] shows, in particular, that the small quantum orbifold cohomology $Q H_{\text {orb }}^{\bullet}(w \mathbb{P})$ has rank $d=\sum w_{i}$ and, in the natural basis, the quantum ordinary differential operator is

$$
P=-t+\prod_{i=0}^{n} w_{i} D\left(w_{i} D-1\right) \cdots\left(w_{i} D-\left(w_{i}-1\right)\right) .
$$

If $H$ is the hypergeometric operator in Equation (11), denote by $\mathcal{M}_{H^{\text {red }}}$ and $\mathcal{M}_{P}$ the $\mathcal{D}$-modules on $\mathbb{C}^{\times}$corresponding to $H^{\text {red }}$ and $P$. The formulae in Kat90, 6.4.2] state that

$$
\mathrm{FT} j_{! *}[d]^{*} \mathcal{M}_{P} \cong j_{! *}[d]^{*} \mathcal{M}_{H^{\text {red }}} \text { and } \quad \mathrm{FT} j_{!_{*}}[d]^{*} \mathcal{M}_{H^{\mathrm{red}}} \cong j_{! *}[d]^{*} \mathcal{M}_{P}
$$

In other words, the $d$-th Kummer pull-backs of the $\mathcal{D}$-modules $\mathcal{M}_{P}$ and $\mathcal{M}_{H^{\text {red }}}$ are each other's Fourier transforms. This is a weak statement of mirror symmetry between $w \mathbb{P}$ and the pencil $f: Y \rightarrow \mathbb{C}^{\times}$. See Gol01, where $\mathcal{M}_{P}$ and $\mathcal{M}_{H^{\text {red }}}$ are called, respectively, the RiemannRoch and the anticanonical Riemann-Roch $\mathcal{D}$-modules; the article AKO contains a much deeper discussion of the case of weighted projective planes.

\subsection{Calabi-Yau weighted hypersurfaces}

The following notions are standard and well-known. 
Definition 1.9. 1. We say that a weighted projective space $w \mathbb{P}=$ $\mathbb{P}\left(w_{0}, \ldots, w_{n}\right)$ is well-formed if $\operatorname{hcf}\left(w_{0}, \ldots, \widehat{w_{i}}, \ldots, w_{n}\right)=1$ for all $i$.

2. A weighted hypersurface $X_{e} \subset w \mathbb{P}$ of degree $e$ is well-formed IF00 if $X_{e}$ does not contain any codimension 2 coordinate subspace $\left(x_{i}=x_{j}=0\right)$.

3. We say that $X_{e}$ is quasismooth if it is well-formed and the affine cone over $X_{e}$ is nonsingular.

Remark 1.10. It is shown in [F00] that, when $X_{e}$ is well-formed, the usual adjunction formula $K_{X_{e}}=K_{w \mathbb{P}}+X_{e \mid X_{e}}=\mathcal{O}_{X_{e}}(e-d)$ holds.

Definition 1.11. Bat94, Definition 4.1.8] A $n$-dimensional projective variety $Y$ is Calabi-Yau if $Y$ has canonical singularities, $K_{Y} \cong \mathcal{O}_{Y}$ (in particular, $Y$ is Gorenstein), and $H^{i}\left(Y, \mathcal{O}_{Y}\right)=(0)$ for $i=1, \ldots, n-1$. A $K 3$ surface is a 2-dimensional Calabi-Yau variety.

(We recall the definition of canonical singularities in Section 3.2 below.)

Lemma 1.12. A quasismooth weighted hypersurface $X_{d}$ of degree $d=$ $\sum w_{i}$ in $\mathbb{P}\left(w_{0}, \ldots, w_{n}\right)$ is a Calabi-Yau variety.

Proof. Indeed, by Remark 1.10, $K_{X} \cong \mathcal{O}_{X}$; in particular, $X$ has Gorenstein singularities. Because $X$ is quasismooth, it has cyclic quotient singularities; it follows that $X$ has Gorenstein rational, and hence canonical singularities. Finally, by Dol82, Theorem 3.2.4], $H^{i}\left(X, \mathcal{O}_{X}\right)=(0)$ for $i=1, \ldots, n-1$.

In the case of surfaces, a general member $X_{d} \subset w \mathbb{P}^{3}$ is Calabi-Yau if and only if it is quasismooth:

Theorem 1.13. A general hypersurface $X_{d} \subset \mathbb{P}\left(w_{0}, \ldots, w_{3}\right)$ is a wellformed K3 surface if and only if it is quasismooth.

Theorem 1.13 is proved in Section 3 .

Remark 1.14. The above fails completely in higher dimensions. For instance, in the list of Kreuzer and Skarke ${ }^{2}$, there are 184,026 CalabiYau weighted hypersurfaces in dimension 3; of these, only 7,555 are quasismooth $^{3}$; a typical example is $X_{99} \subset \mathbb{P}(15,18,19,20,27)$. (We are grateful to M. Reid for this remark.)

\footnotetext{
${ }^{2}$ see http://hep.itp.tuwien.ac.at/ kreuzer/CY/

${ }^{3}$ see http://pcmat12.kent.ac.uk/grdb/
} 
The discussion above suggests that Calabi-Yau hypersurfaces $X_{d} \subset$ $w \mathbb{P}$ are mirror-dual to the fibres of the pencil $f: Y \rightarrow \mathbb{C}^{\times}$described by Equation (2). We investigate some crude geometric consequences of this statement and we show that it is compatible with Batyrev's Bat94 view of mirror symmetry.

Theorem 1.15. Fix a well-formed weighted projective space $w \mathbb{P}=$ $\mathbb{P}\left(w_{0}, \ldots, w_{n}\right)$ and let $f: Y \rightarrow \mathbb{C}^{\times}$be the pencil described by Equation (2). A well-formed general hypersurface $X_{d} \subset w \mathbb{P}$ of degree $d=\sum w_{i}$ is Calabi-Yau if and only if a general fibre $Y_{t}=f^{-1}(t)$ is birational to a Calabi-Yau variety.

Remark 1.16. The proof of Theorem 1.15, which is carried out in $\$ 3$ below, is based on showing that, in the Calabi-Yau case, there are a resolution of $X_{d}$ and a nonsingular completion of $Y_{t}$ which are mirror-dual (nonsingular) Calabi-Yau toric hypersurfaces in the sense of Bat94.

Presumably, it is possible to enumerate orbifold rational curves on a quasismooth Calabi-Yau weighted hypersurface in terms of the polarised variation of Hodge structure $\operatorname{gr}_{n-1}^{W} R^{n-1} f_{!} \mathbb{Z}_{Y}$.

As we already remarked in Corollary [1.6] $w \mathbb{P}$ has canonical singularities if and only if $h_{c}^{n-1,0}\left(Y_{t}\right)=1$. For $n=3$, there are exactly 104 weighted projective 3 -spaces $\mathbb{P}\left(w_{0}, \ldots, w_{3}\right)$ with canonical singularities (as can be checked by writing a small computer program). The corresponding sets of weights are the famous 95 of [F00, §13.3, pp. 138-140] plus the additional nine of Table 1 In the case of one of

\begin{tabular}{cccc}
$w_{0}$ & $w_{1}$ & $w_{2}$ & $w_{3}$ \\
\hline 1 & 5 & 6 & 8 \\
\hline 1 & 4 & 7 & 9 \\
\hline 2 & 5 & 8 & 9 \\
\hline 1 & 5 & 8 & 14 \\
\hline 3 & 7 & 8 & 10 \\
\hline 4 & 7 & 9 & 10 \\
\hline 5 & 8 & 9 & 11 \\
\hline 3 & 7 & 8 & 18 \\
\hline 5 & 8 & 9 & 22
\end{tabular}

Table 1: The additional nine sets of weights 
the additional nine sets of weights, a general surface $X_{d} \subset w \mathbb{P}$ is wellformed but has non-canonical singularities; nevertheless, $\operatorname{gr}_{2}^{W} R^{2} f ! \mathbb{Z}_{Y}$ is a polarised variation of Hodge structure of weight 2 and $h^{2,0}=1$. It would be interesting to study these PVHS in greater detail. In particular, it seems likely that each fibre of the local system can be embedded in the K3 lattice and hence, by Torelli, it is the transcendental lattice of a K3 surface. However, the local system as a whole may not be the PVHS attached to the variation of transcendental lattice in a pencil of K3 surfaces, possibly because the global monodromy acts nontrivially on the discriminant group and hence does not lift to an action on the K3 lattice.

Theorem 1.17. In the additional nine cases, $Y_{t}$ is birational to an elliptic surface of Kodaira dimension 1.

Theorems 1.13 1.15 and 1.17 are proved in Section 3

\section{Proof of Theorems 1.1 and 1.3}

Step 1 Recall the pencil of Equation (2):

$$
Y=\left\{\begin{array}{l}
\prod_{i=0}^{n} y_{i}^{w_{i}}=t \\
\sum_{i=0} y_{i}=1
\end{array} \quad \subset \mathbb{C}^{\times n+1} \times \mathbb{C}^{\times}\right.
$$

and $f=\operatorname{pr}_{2}: Y \rightarrow \mathbb{C}^{\times}$. In the notation of the Introduction:

$$
N=\mathbb{Z}\left(\frac{w_{0}}{d}, \ldots, \frac{w_{n}}{d}\right)+\mathbb{Z}^{n+1},
$$

$I=\mathbb{R}_{+}^{n+1} \subset N_{\mathbb{R}}$ is the positive orthant, $l: N \rightarrow \mathbb{Z}$ is the linear form of Equation (3), $a: \mathbb{Z} / d=N / \mathbb{Z}^{n+1} \rightarrow[0, n] \cap \mathbb{Z}$ is the age function,

$$
(\mathbb{Z} / d)^{0}=\left(N / \mathbb{Z}^{n+1}\right)^{0}=\left\{0 \leq k<d \mid d \text { divides no } k w_{i}\right\}
$$

and $a_{j}^{s}=\operatorname{card} a^{-1}(j) \cap(\mathbb{Z} / d)^{0}$.

In this step, we show that

$$
h^{n-j, j-1}\left(H_{c}^{n-1} Y_{t}\right)=a_{j}^{s} .
$$

Definition 2.1. The Landau-Ginzburg pencil or LG pencil is the pullback $f^{\prime}: Y^{\prime} \rightarrow \mathbb{C}^{\times}$of $f: Y \rightarrow \mathbb{C}^{\times}$by the covering $t=1 / u^{d}: \mathbb{C}^{\times} \rightarrow \mathbb{C}^{\times}$; after the change of coordinates $y_{i} \mapsto y_{i} / u$, we have:

$$
Y^{\prime}=\left(\prod_{i=0}^{n} y_{i}^{w_{i}}=1\right) \subset \mathbb{C}^{\times n+1} \quad \text { and } \quad f^{\prime}=u=\sum_{i=0}^{n} y_{i} .
$$


In what follows, it is convenient to work with the Landau-Ginzburg pencil $u: Y^{\prime} \rightarrow \mathbb{C}^{\times}$; as in the Introduction, we think of the affine hyperplane $N_{1}=l^{-1}(1) \cap N$ as a lattice by choosing $\left(w_{0} / d, \ldots, w_{n} / d\right)=$ e as the origin; the projection $\mathbf{w} \mapsto \mathbf{w}-l(\mathbf{w}) \mathbf{e}+\mathbf{e}$ identifies $N_{1}$ with the lattice of characters of the $n$-dimensional torus

$$
\mathbb{T}^{n}=Y^{\prime}=\left\{\left(a_{0}, \ldots, a_{n}\right) \in \mathbb{C}^{\times n+1} \mid \prod a_{i}^{w_{i}}=1\right\} \subset \mathbb{C}^{n+1} .
$$

Denote by $\Delta=I \cap N_{1 \mathbb{R}}$ the simplex generated by the vectors $\mathbf{e}_{i}$. We may identify the Landau-Ginzburg fibre $Y_{u}^{\prime}=Y_{1 / t^{d}}$ with the hypersurface

$$
\left(-u \mathbf{e}+\sum \mathbf{e}_{i}=0\right) \subset \mathbb{T}^{n}=Y^{\prime}
$$

in this description, it is clear that $\Delta$ is the Newton polyhedron of $Y_{u}^{\prime}$, hence $Y_{u}^{\prime}$ is (tautologically) Newton-regular (see Definition 3.11).

We use the notation and results of [DK86, $\S 4$ and $\S 5]$; in particular, we use the Poincaré series

$$
L_{\Delta}^{*}(t)=\sum_{k \geq 0} \ell^{*}(k \Delta) t^{k}
$$

where $\ell^{*}(k \Delta)$ is the number of lattice points strictly inside $k \Delta$. It is shown in [DK86, §4] that

$$
P_{\Delta}(t)=(1-t)^{n+1} L_{\Delta}^{*}(t)=\sum_{k=1}^{n} \varphi_{k}(\Delta) t^{k}
$$

is a polynomial. The statements DK86, 3.11, 4.4, 5.6] imply that:

$$
\begin{aligned}
& h^{n-k, k-1}\left(H_{c}^{n-1} Y_{t}\right)= \\
& =\varphi_{k}(\Delta)-\sum_{\operatorname{dim} \Gamma=n-1} \varphi_{k-1}(\Gamma)+\sum_{\operatorname{dim} \Gamma=n-2} \varphi_{k-2}(\Gamma)-\cdots
\end{aligned}
$$

We consider the locally closed stratification of $I$ with strata corresponding to intersections of coordinate hyperplanes and their translations by lattice vectors. For $\mathbf{w} \in N \cap I$, we define the content $c(\mathbf{w})$ :

$$
c(\mathbf{w})=k \quad \text { if } \mathbf{w} \text { lies on the stratum of codimension } k \text {. }
$$

Let $B=\left\{b=\left(b_{0}, \ldots, b_{n}\right) \subset \mathbb{R}^{n} \mid\right.$ all $\left.0 \leq b_{i}<1\right\}$ be the unit box; note that:

$$
a_{k}^{s}=\operatorname{card}\{\mathbf{w} \in N \cap B \mid l(\mathbf{w})=k \text { and } c(\mathbf{w})=0\} .
$$


We count using the exclusion-inclusion principle over strata of codimension $k$ (the notation is self-explanatory):

$$
\begin{aligned}
\frac{1}{(1-t)^{n+1}} \sum_{k=1}^{n} a_{k}^{s} t^{k} & =\sum_{\substack{\mathbf{w} \in N \cap I \\
c(\mathbf{w})=0}} t^{l(\mathbf{w})}= \\
& =\sum_{\mathbf{w} \in N \cap I} t^{l(\mathbf{w})}-\sum_{\substack{\mathbf{w} \in N \cap I \\
c(\mathbf{w}) \geq 1}} t^{l(\mathbf{w})}= \\
=L_{\Delta}^{*}(t)-\frac{t}{1-t} & \sum_{\operatorname{codim} F=1} L_{F}^{*}(t)+\frac{t^{2}}{(1-t)^{2}} \sum_{\operatorname{codim} F=2} L_{F}^{*}(t)-\cdots
\end{aligned}
$$

Therefore:

$$
\begin{aligned}
& \sum a_{k}^{s} t^{k}= \\
& =(1-t)^{n+1} L_{\Delta}^{*}(t)-t(1-t)^{n} \sum_{\operatorname{codim} F=1} L_{F}^{*}(t)+ \\
& +t^{2}(1-t)^{n-1} \sum_{\operatorname{codim} F=2} L_{F}^{*}(t)-\cdots= \\
& =P_{\Delta}(t)-t \sum_{\operatorname{codim} F=1} P_{F}(t)+t^{2} \sum_{\operatorname{codim} F=2} P_{F}(t)-\cdots= \\
& =\sum_{k=1}^{n} h^{n-k, k-1} t^{k} .
\end{aligned}
$$

Step 2 The proof of Gol01, Theorem 3.5.1] shows that the irreducible $\ell$-adic local system $\mathbb{V}_{\mathbb{Q}_{\ell}}$ is contained in $\operatorname{gr}_{n-1}^{W} R^{n-1} f_{!} \mathbb{Q}_{\ell Y}$ for all $\ell$; it follows that $\mathbb{V}_{\mathbb{R}}$ is a direct summand of $\operatorname{gr}_{n-1}^{W} R^{n-1} f_{!} \mathbb{R}_{Y}$; summing the $h^{n-k, k-1}$ determined in Step 1 we calculate the rank of $\operatorname{gr}_{n-1}^{W} R^{n-1} f_{!} \mathbb{R}_{Y}$ and conclude that the two local systems have the same rank; therefore, they must coincide. This proves Theorem 1.1 and the calculation in Step 1 then proves Theorem 1.3.

\section{Proof of Theorems 1.13, 1.15, 1.17}

\subsection{Toric varieties}

We use freely the language of toric varieties. This section is here to fix our notation; it is not an introduction to toric varieties. 
Toric varieties If $\mathbb{T}$ is the $n$-dimensional torus, we denote by $M=\operatorname{Hom}\left(\mathbb{T}, \mathbb{C}^{\times}\right)$and $N=\operatorname{Hom}\left(\mathbb{C}^{\times}, \mathbb{T}\right)$ the lattices of monomials (characters of $\mathbb{T}$ ) and weights (one-parameter subgroups of $\mathbb{T}$ ).

We denote by $\mathbb{P}_{\Sigma}$ the toric variety associated to a rational polyhedral fan $\Sigma \subset N_{\mathbb{R}}$. We mostly work with complete fans, that is, proper toric varieties.

Weil and Cartier divisors and sheaves We denote by $\Sigma^{(1)}$ the set of primitive integer generators of the 1-dimensional cones of $\Sigma$; if $\mathbf{v} \in \Sigma^{(1)}, D_{\mathbf{v}} \subset \mathbb{P}_{\Sigma}$ denotes the corresponding prime divisor; for example, the canonical divisor is

$$
K=-\sum_{\mathbf{v} \in \Sigma^{(1)}} D_{\mathbf{v}}
$$

Monomials $\mathbf{m} \in M$ are rational function on $\mathbb{P}_{\Sigma}$ and

$$
\operatorname{div} \mathbf{m}=\sum_{\mathbf{v} \in \Sigma^{(1)}}\langle\mathbf{m}, \mathbf{v}\rangle D_{\mathbf{v}} .
$$

An integer (rational) piecewise linear function $\varphi: N_{\mathbb{R}} \rightarrow \mathbb{R}$ which is linear on the cones of $\Sigma$ gives rise to a Cartier $(\mathbb{Q}$-Cartier) divisor

$$
D=\sum_{\mathbf{v} \in \Sigma^{(1)}} \varphi(\mathbf{v}) D_{\mathbf{v}}
$$

on $\mathbb{P}_{\Sigma}$. The divisor $D$ is nef (ample) if and only if $\varphi$ is convex (strictly convex). If $D=\sum_{\mathbf{v} \in \Sigma^{(1)}} d_{\mathbf{v}} D_{\mathbf{v}}$ is a Weil divisor, we denote by $\mathcal{O}(D)$ the sheaf of rational functions $f$ such that $\operatorname{div} f \geq-D$. When we view a rational function $f \in \mathbb{C}[M]$ as a rational section of $\mathcal{O}(D)$, the divisor of zeros and poles of $f$ is $\operatorname{div} f+D$. A monomial basis of $H^{0}\left(\mathbb{P}_{\Sigma}, \mathcal{O}(D)\right)$ is

$$
\left\{\mathbf{m} \in M \mid\langle\mathbf{m}, \mathbf{v}\rangle \geq-d_{\mathbf{v}} \text { for all } \mathbf{v} \in \Sigma^{(1)}\right\} \subset M .
$$

Polyhedra The datum $(\Sigma, \varphi)$ of a fan $\Sigma$ and a strictly convex integer piecewise linear function $\varphi: N_{\mathbb{R}} \rightarrow \mathbb{R}$ linear on cones is equivalent to the datum of an integer strictly convex polyhedron $Q \subset M_{\mathbb{R}}$ containing the origin. Given $(\Sigma, \varphi)$, we set

$$
Q=\left\{\mathbf{m} \in M \mid\langle\mathbf{m}, \mathbf{v}\rangle \geq-\varphi(\mathbf{v}) \text { for all } \mathbf{v} \in \Sigma^{(1)}\right\} \subset M .
$$




\section{Toric hypersurfaces}

Definition 3.1. A toric hypersurface $Z_{f}=(f=0) \subset X_{\Sigma}$ is Newtonregular if it meets transversally all the toric strata.

Remark 3.2. Consider a convex integer polyhedron $0 \in Q \subset M_{\mathbb{R}}$ and let $\left(\mathbb{P}_{Q}, L_{Q}\right)$ be the corresponding polarised toric variety. Consider a set of lattice points $S \subset Q \cap M$ and the linear system $\mathcal{D}=|S| \subset$ $\mathbb{P} H^{0}\left(\mathbb{P}_{Q}, L_{Q}\right)$ that they generate. Then, a general member of $\mathcal{D}$ is Newton-regular if and only if $Q$ is the convex envelope of $S$.

\subsection{Discrepancies and canonical singularities}

We recall the definition of discrepancy of a valuation and the notion of canonical singularities.

Definition 3.3. $\quad 1$. Let $X$ be a normal variety of dimension $n$. We denote by $k(X)$ the field of rational functions on $X$. A rational differential $\omega \in \Omega_{k(X)}^{n}$ determines a canonical divisor of $X$ :

$$
K_{X}=\operatorname{div}_{X} \omega .
$$

2. A discrete rank 1 valuation $\nu$ of $k(X)$ is geometric (sometimes called divisorial in the valuation theory literature) if it has a uniformisation, that is, a pair $E \subset Y$ of a normal variety $Y$ with $k(Y)=k(X)$, and a prime divisor $E \subset Y$ such that $\nu=$ mult $_{E}$ measures multiplicity along $E$. Abusing notation, we often make no distinction between the valuation $\nu$ and the divisor $E$. We say that $\nu$ has centre on $X$, or that it is a valuation of $X$ or on $X$, if the obvious rational map $f: Y \rightarrow X$ is regular at $E$; the centre of $\nu$ (or $E$ ) is the scheme-theoretic point $f(E)=$ centre $_{X} E$. We say that $\nu$ has small centre if centre $X E$ has codimension $\geq 2$.

3. Let $\nu$ be a geometric valuation with centre on $X$ and uniformisation $f: E \subset Y \rightarrow X$. If $K_{X}$ is $\mathbb{Q}$-Cartier, we define the discrepancy of $\nu$ as:

$$
a(\nu)=\operatorname{mult}_{E}\left(K_{Y}-f^{*} K_{X}\right)
$$

Note that, because $f$ is birational, $k(X)=k(Y)$; in the formula, it is understood that $K_{Y}=\operatorname{div}_{Y} \omega$. On the other hand, $f^{*} K_{X}$ denotes the pull-back of a $\mathbb{Q}$-Cartier divisor. It is easy to see that the discrepancy does not depend on the choice of uniformisation and the differential $\omega$. 
4. Consider now a pair $(X, B)$ of a normal variety $X$ and a boundary $B$; that is, $B=\sum b_{i} B_{i} \subset X$ is a $\mathbb{Q}$-divisor with prime components $B_{i}$ and $0<b_{i} \leq 1$. We say that $B$ is a strict boundary if all $b_{i}<1$. If $\nu$ is a geometric valuation of $X$ with uniformisation $f:(E \subset Y) \rightarrow X$, and $K_{X}+B$ is $\mathbb{Q}$-Cartier, we define the discrepancy of $\nu$ with respect to the pair $(X, B)$ as:

$$
a(\nu, B)=\operatorname{mult}_{E}\left(K_{Y}-f^{*}\left(K_{X}+B\right)\right) .
$$

Definition 3.4. $\quad$ 1. $X$ has canonical (terminal) singularities if $K_{X}$ is $\mathbb{Q}$-Cartier and every geometric valuation of $X$ has $\geq 0(>0)$ discrepancy.

2. The pair $(X, B)$ has $k l t$, or Kawamata log terminal, singularities if $K_{X}+B$ is $\mathbb{Q}$-Cartier and every geometric valuation $\nu$ of $X$ has $a(\nu, B)>-1$.

3. The pair $(X, B)$ has plt, or purely log terminal, singularities if $K_{X}+B$ is $\mathbb{Q}$-Cartier and every geometric valuation $\nu$ with small centre on $X$ has $a(\nu, B)>-1$.

4. The pair $(X, B)$ has canonical (terminal) singularities if $K_{X}+B$ is $\mathbb{Q}$-Cartier and every geometric valuation $\nu$ with small centre on $X$ has $a(\nu, B) \geq 0(>0)$.

5. $X$ has pseudo-terminal singularities if it has canonical singularities and $a(\nu)>0$ for every geometric valuation $\nu$ whose centre centre $_{X} \nu$ has codimension $\geq 3$ in $X$.

Remark 3.5. If $(X, B)$ has klt singularities, then $B$ is a strict boundary. It can be shown that if $(X, B)$ has plt singularities, then

$$
\lfloor B\rfloor=\sum_{b_{i}=1} B_{i}
$$

is a disjoint union of normal components.

Definition 3.6. A good resolution of a pair $(X, \mathcal{D})$ of a variety $X$ and a linear system $\mathcal{D}$ on $X$ is a proper birational morphism $f: Y \rightarrow X$ where:

1. $Y$ is nonsingular and the exceptional set $\operatorname{Exc} f$ is a simple normal crossing divisor, and

2. the birational transform $\mathcal{D}^{\prime}$ is a base point free linear system. 
Definition 3.7. If $X$ is a variety and $\mathcal{D}$ a linear system on $X$, we say that the pair $(X, \mathcal{D})$ has canonical (terminal) singularities if there is a good resolution $f: X \rightarrow Y$ with exceptional divisors $E_{i} \subset Y$ such that

$$
K_{Y}+\mathcal{D}^{\prime}=f^{*}\left(K_{X}+\mathcal{D}\right)+\sum a_{i} E_{i}
$$

with all $a_{i} \geq 0(>0)$.

Remark 3.8. If the pair $(X, \mathcal{D})$ has canonical (terminal) singularities, and $D \in \mathcal{D}$ is a general member, then $(X, D)$ has canonical (terminal) singularities. The converse is often not true.

We refer the reader to Rei87 for an accessible introduction to canonical singularities. It is easy to see that $X$ has canonical singularities if $a(\nu) \geq 0$ for all $\nu$ which are uniformised by a fixed resolution $f: Y \rightarrow X$. When $\mathbb{P}$ is a toric variety, then $\mathbb{P}$ has a toric resolution; in this case, it is enough to test toric valuations associated to primitive vectors in the lattice $N$.

\subsection{Proof of Theorem 1.15}

Let $\mathbb{T}$ be the $n$-dimensional torus; denote by $M=\operatorname{Hom}\left(\mathbb{T}, \mathbb{C}^{\times}\right)$and $N=\operatorname{Hom}\left(\mathbb{C}^{\times}, \mathbb{T}\right)$ the lattices of monomials and weights. The space $w \mathbb{P}^{n}=\mathbb{P}\left(w_{0}, \ldots, w_{n}\right)$ is a toric variety corresponding to a choice of primitive integer vectors $\mathbf{e}_{i} \in N$ satisfying the relation $\sum w_{i} \mathbf{e}_{i}=\mathbf{0}$. In what follows, we denote by $\Delta \subset N_{\mathbb{R}}$ the simplex generated by the $\mathbf{e}_{i}$.

Assume that a general $X_{d} \subset w \mathbb{P}^{n}$ is a well-formed Calabi-Yau. Consider the dual simplex

$$
\Delta^{*}=\left\{\mathbf{m} \in M_{\mathbb{R}} \mid \text { all }\left\langle\mathbf{m}, \mathbf{e}_{\mathbf{i}}\right\rangle \geq-1\right\} \subset M_{\mathbb{R}} ;
$$

note that $\Delta^{*}$ has rational not necessarily integer vertices. The integer points in $\Delta^{*}$ are a basis of $H^{0}\left(w \mathbb{P},-K_{w \mathbb{P}}\right)$. Consider the integer polyhedron $Q \subset M$ generated by the integer points in $\Delta^{*}$, and the associated polarised toric variety $\left(\mathbb{P}_{Q}, L_{Q}\right)$; by construction, $L=-K_{\mathbb{P}_{Q}}$ and the proper transform of a general $X_{d} \in\left|\mathcal{O}_{w \mathbb{P}}(d)\right|$ is a Newton-regular Calabi-Yau variety $X^{\prime} \subset\left|-K_{\mathbb{P}_{Q}}\right|$. By [Bat94, Theorem 4.1.9], $Q$ is a reflexive polyhedron, and so is the dual polyhedron $P=Q^{*} \subset N$. Denote by $\mathbb{P}_{P}$ the associated dual toric variety under the dual torus; by construction, $\Delta \subset P$; therefore, the proper transform

$$
Y_{u}^{\prime \prime}=\left(-u \mathbf{0}+\sum_{i=0}^{n} \mathbf{e}_{i}=0\right) \in\left|-K_{\mathbb{P}_{P}}\right|
$$


of the Landau-Ginzburg pencil is a pencil of anticanonical sections of $\mathbb{P}_{P}$. The integer points in $P$ form a basis of $H^{0}\left(\mathbb{P}_{P},-K_{\mathbb{P}_{P}}\right)$; more precisely, an integer vector $\mathbf{v} \in P$ is a monomial on the dual torus; when viewed as a section of $-K$, its divisor of zeros is

$$
Z(\mathbf{v})=\operatorname{div} \mathbf{v}-K \sim-K
$$

(here $-K$ is the standard anticanonical divisor of $\mathbb{P}_{P}$, that is, the sum of all the codimension 1 strata with multiplicity 1). Batyrev Bat94, Theorem 4.1.9] states that a general element of $\left|-K_{\mathbb{P}_{P}}\right|$ is CalabiYau; we claim that a general $Y_{u}^{\prime \prime}$ is also Calabi-Yau; in other words, we are saying that $Y_{u}^{\prime \prime}$ is a projective birational Calabi-Yau model of the Landau-Ginzburg fibre $Y_{u}^{\prime}$. This is the same as saying that a general member of the linear system

$$
\mathcal{D}=\left|Z\left(\mathbf{e}_{i}\right), Z(\mathbf{0})\right|
$$

is Calabi-Yau (absorb degrees of freedom using the torus action); for this, it is enough to show that the pair $\left(\mathbb{P}_{P}, \mathcal{D}\right)$ has canonical singularities; that is, for all valuations $\nu$ with small centre on $\mathbb{P}_{P}$ :

$$
a(\nu, \mathcal{D})=a\left(\nu, K_{\mathbb{P}_{P}}\right)-\text { mult }_{\nu} \mathcal{D} \geq 0 .
$$

It suffices to consider toric valuations corresponding to primitive integer vectors $\mathbf{m} \in M \backslash\{\mathbf{0}\}$; then:

$$
a\left(\mathbf{m}, K_{\mathbb{P}_{P}}\right)=-1-\text { mult }_{\mathbf{m}} K
$$

and

$$
\text { mult }_{\mathbf{m}} \mathcal{D}=- \text { mult }_{\mathbf{m}} K+\min \left\{\left\langle\mathbf{m}, \mathbf{e}_{i}\right\rangle\right\}
$$

therefore, the statement follows from the claim that

$$
-1 \geq \min \left\{\left\langle\mathbf{m}, \mathbf{e}_{i}\right\rangle\right\} .
$$

This, however, is obvious: indeed, if it fails, that is precisely saying that all $\left\langle\mathbf{m}, \mathbf{e}_{i}\right\rangle>-1$, that is, $\mathbf{m}$ is strictly inside of $\Delta^{*}$. The assumption that $X_{d}$ is a well-formed Calabi-Yau means exactly that $\mathbf{0}$ is the only lattice point strictly inside of $\Delta^{*}$.

Vice-versa, assume that a general member $Y_{u}^{\prime}$ is birational to a Calabi-Yau. We have already observed in the proof of Theorem 1.1 that $Y_{u}^{\prime}$ is Newton-regular. By [sh99, we can construct a (partial) resolution and minimal model $\left(\mathbb{P}_{\Sigma}, Y_{u}^{\prime \prime}\right)$ where the birational transform $Y_{u}^{\prime \prime} \in\left|-K_{\mathbb{P}_{\Sigma}}\right|$ is a Newton-regular Calabi-Yau. Again by Bat94, 
Theorem 4.1.9], the toric variety $\mathbb{P}_{\Sigma}$ is the toric variety $\mathbb{P}_{P}$ attached to a reflexive integer polyhedron $P \subset N$ and it is obvious from the construction that $\Delta \subset P$. If now $Q=P^{*}$ is the dual reflexive polyhedron, and $\mathbb{P}_{Q}$ is the associated dual toric variety, the inclusion $\Delta \subset P$ gives a birational morphism $f: \mathbb{P}_{Q} \rightarrow w \mathbb{P}$. By Batyrev, a general member $\tilde{X} \in\left|-K_{\mathbb{P}_{Q}}\right|$ is a Newton-regular Calabi-Yau; it follows that $X=f(\widetilde{X})$ is Calabi-Yau.

\subsection{Proof of Theorem 1.13}

In the proof, we need the following result of Shokurov and Kollár Kol92 Theorem 17.6]. Let $X$ a normal variety, $B=\sum b_{i} B_{i}$ a strict boundary, and $S \subset X$ a normal subvariety of codimension 1 not contained in the support of $B$. As explained in [Kol92, Ch. 16], if $K+S+B$ is $\mathbb{Q}$-Cartier, there is a naturally defined $\mathbb{Q}$-divisor $B_{S}=\operatorname{Diff}_{S} B$ such that the adjunction formula

$$
\left(K_{X}+S+B\right)_{S}=K_{S}+B_{S}
$$

holds.

Theorem 3.9 (Inversion of adjunction). The pair $(X, S+B)$ has plt singularities in a neighbourhood of $S$ if and only if the pair $\left(S, B_{S}\right)$ has klt singularities.

Proof of Theorem 1.13. If $X=X_{d} \subset w \mathbb{P}$ is a well-formed K3, then, by Theorem 3.9 with $S=X$ and $B=0$, the pair $(w \mathbb{P}, X)$ has plt singularities hence $\left(K_{w \mathbb{P}}+X\right.$ is Cartier, so all discrepancies $a(E, X)$ are integers!) canonical singularities in a neighbourhood of $X$; it follows that $w \mathbb{P}$ has pseudo-terminal singularities along $X$; on the other hand, $w \mathbb{P}$ has Gorenstein quotient hence canonical singularities outside of $X$; therefore, $w \mathbb{P}$ has canonical singularities, hence it belongs to the list of 104 . We can check, by direct inspection, that if a general $X_{d}$ is a $\mathrm{K} 3$, then it is a quasismooth K3: this is known in the case of the 95; in the additional nine cases, we verify that $X_{d}$ is not a K3 surface.

\subsection{Proof of Theorem 1.17}

The proof is an elementary verification. Recall the notation and construction of the Landau-Ginzburg pencil:

$$
Y^{\prime}=\left(\prod_{i=0}^{n} y_{i}^{w_{i}}=1\right) \subset \mathbb{C}^{\times n+1} \quad \text { and } \quad u=\sum_{i=0} y_{i}: Y^{\prime} \rightarrow \mathbb{C}^{\times} .
$$


As usual we write $N=\mathbb{Z}\left(w_{0} / d, \ldots, w_{n} / d\right)+\mathbb{Z}^{n+1}$ and $I=\mathbb{R}_{+}^{n+1} \subset N_{\mathbb{R}}$; we denote by $\mathbf{e}_{i}$ the standard basis vectors of $\mathbb{Z}^{n+1}$ and

$$
\mathbf{e}=\left(\frac{w_{0}}{d}, \ldots, \frac{w_{n}}{d}\right) \in N
$$

The linear function $l: N \rightarrow \mathbb{Z}$ of Equation (3) defines a grading on $\mathbb{C}[N]$ and the Landau-Ginzburg fibre $Y_{u}^{\prime} \subset Y^{\prime}$ naturally compactifies to a hypersurface in a simplicial toric variety:

$$
\overline{Y_{u}^{\prime}}=\left(-u \mathbf{e}+\sum \mathbf{e}_{i}=0\right) \subset \mathbb{P}=\operatorname{Proj} \mathbb{C}[N \cap I] .
$$

We use this compactification to study the Landau-Ginzburg pencil $u: Y^{\prime} \rightarrow \mathbb{C}^{\times}$. To display information in a human-readable format, it is convenient, though not necessary and sometimes misleading, to realise the simplicial toric variety as a quotient of a weighted projective space by the action of a product of cyclic groups as in the following:

Lemma 3.10. Let $b_{i}=\operatorname{hcf}\left(w_{i}, d\right)$ and write $w_{i}=u_{i} b_{i}, d=d_{i} b_{i}$ with $\operatorname{hcf}\left(u_{i}, d_{i}\right)=1$. Then (in the notation of the preceding discussion)

$$
\mathbb{P}=\mathbb{P}\left(b_{0}, \ldots, b_{n}\right) / G
$$

where $G$ is a product of (at most) $n-1$ cyclic groups acting diagonally and faithfully. Writing $\tilde{\mathbf{e}}_{i}=\mathbf{e}_{i} / d_{i}$ and $\tilde{N}=\sum \mathbb{Z} \tilde{\mathbf{e}}_{i}$, there are split exact sequences:

$$
\begin{gathered}
0 \rightarrow \operatorname{Hom}\left(\tilde{N} / N, \mathbb{C}^{\times}\right) \rightarrow \prod \mu_{d_{i}} \rightarrow \mu_{d} \rightarrow 0, \\
0 \rightarrow \mu_{d} \rightarrow \operatorname{Hom}\left(\tilde{N} / N, \mathbb{C}^{\times}\right) \rightarrow G \rightarrow 0 .
\end{gathered}
$$

Proof. The proof is an easy exercise. It is clear that $\mathbb{Z}^{n+1} \subset N \subset \widetilde{N}$ and that $\mathbb{P}$ is the quotient of

$$
\mathbb{P}\left(b_{0}, \ldots, b_{n}\right)=\operatorname{Proj} \mathbb{C}[\tilde{N}]
$$

by the subgroup $\operatorname{Hom}\left(\widetilde{N} / N, \mathbb{C}^{\times}\right)$consisting of the elements of $\prod \mu_{d_{i}}=$ $\operatorname{Hom}\left(\tilde{N} / \mathbb{Z}^{n+1}, \mathbb{C}^{\times}\right)$that fix $\mathbf{e} \in N$; this group does not act faithfully on $\mathbb{P}\left(b_{0}, \ldots, b_{n}\right)$; dividing out by diagonal elements yields $G$.

Proof of Theorem 1.17. The proof is a calculation summarised in Table 2. Here, we explain what the table says and discuss the first entry in detail; the reader with sufficient motivation can perform the calculation in the remaining eight cases. 
The first column of Table 2 lists the nine weights of Table 1 the second column gives the equation $F$ of the pencil element $\overline{Y_{u}^{\prime}}=(F=$ $0)$ in the natural coordinates $z_{0}, \ldots, z_{3}$ of $\mathbb{P}\left(b_{0}, \ldots, b_{3}\right)$ corresponding to the vectors $\tilde{\mathbf{e}}_{0}, \ldots \tilde{\mathbf{e}}_{3}$ described in Lemma 3.10 the third column expresses the simplicial toric variety $\mathbb{P}=\overline{Y^{\prime}}$ as $\mathbb{P}\left(b_{0}, \ldots, b_{3}\right) / G$ and identifies the group $G$; the fourth column gives the unique $G$-invariant differential

$$
\omega=\operatorname{res} \frac{g(z) \Omega}{\overline{Y_{u}^{\prime}}} \frac{g}{F}
$$

on $\overline{Y_{u}^{\prime}}$ where $\Omega=\sum(-1)^{i} z_{i} d z_{0} \cdots \widehat{d z_{i}} \cdots d z_{3}$ is the Griffiths differential on $\mathbb{P}\left(b_{0}, \ldots, b_{3}\right)$; finally, the last column lists a monomial $m\left(z_{0}, \ldots, z_{3}\right)$ and the claim is that $m \in N$ is of degree 0 and the rational map

$$
m: \overline{Y_{u}^{\prime}} \rightarrow \mathbb{P}^{1}
$$

is an elliptic fibration expressing $\overline{Y_{u}^{\prime}}$ as an elliptic surface with Kodaira

\begin{tabular}{|c|c|c|c|c|}
\hline weights & $F$ & ambient & $\omega$ & map \\
\hline $1,5,6,8$ & $\begin{array}{r}z_{0}^{20}+z_{1}^{4}+z_{2}^{10}+z_{3}^{5} \\
-u z_{0} z_{1} z_{2}^{3} z_{3}^{2}\end{array}$ & $\mathbb{P}(1,5,2,4) / \mu_{10}$ & $\frac{z_{2}^{2} z_{3} \Omega}{F}$ & $\frac{z_{3}^{2}}{z_{0}^{4} z_{2}^{2}}$ \\
\hline $1,4,7,9$ & $\begin{array}{r}z_{0}^{21}+z_{1}^{21}+z_{2}^{3}+z_{3}^{7} \\
-u z_{0} z_{1}^{4} z_{2} z_{3}^{3}\end{array}$ & $\mathbb{P}(1,1,7,3) / \mu_{21}$ & $\frac{z_{1}^{3} z_{3}^{2} \Omega}{F}$ & $\frac{z_{3}^{3}}{z_{0}^{6} z_{1}^{3}}$ \\
\hline $2,5,8,9$ & $\begin{array}{r}z_{0}^{12}+z_{1}^{24}+z_{2}^{3}+z_{3}^{8} \\
-u z_{0} z_{1}^{5} z_{2} z_{3}^{3}\end{array}$ & $\mathbb{P}(2,1,8,3) / \mu_{12}$ & $\frac{z_{1}^{4} z_{3}^{2} \Omega}{F}$ & $\frac{z_{3}^{3}}{z_{0}^{3} z_{1}^{3}}$ \\
\hline $1,5,8,14$ & $\begin{array}{r}z_{0}^{28}+z_{1}^{28}+z_{2}^{7}+z_{3}^{2} \\
-u z_{0} z_{1}^{5} z_{2}^{2} z_{3}\end{array}$ & $\mathbb{P}(1,1,4,14) / \mu_{14}$ & $\frac{z_{1}^{4} z_{2} \Omega}{F}$ & $\frac{z_{2}^{2}}{z_{0}^{6} z_{1}}$ \\
\hline $3,7,8,10$ & $\begin{array}{r}z_{0}^{28}+z_{1}^{4}+z_{2}^{7}+z_{3}^{14} \\
-u z_{0}^{3} z_{1} z_{2}^{2} z_{3}^{5}\end{array}$ & $\mathbb{P}(1,7,4,2) / \mu_{14}$ & $\frac{z_{0}^{2} z_{2} z_{3}^{4} \Omega}{F}$ & $\frac{z_{2}^{2}}{z_{0}^{4} z_{3}^{2}}$ \\
\hline $4,7,9,10$ & $\begin{array}{r}z_{0}^{15}+z_{1}^{30}+z_{2}^{10}+z_{3}^{3} \\
-u z_{0}^{2} z_{1}^{7} z_{2}^{3} z_{3}\end{array}$ & $\mathbb{P}(2,1,3,10) / \mu_{15}$ & $\frac{z_{0} z_{1}^{6} z_{2}^{2} \Omega}{F}$ & $\frac{z_{2}^{2}}{z_{0}^{4} z_{1}^{4} z_{4}}$ \\
\hline $5,8,9,11$ & $\begin{array}{r}z_{0}^{33}+z_{1}^{33}+z_{2}^{11}+z_{3}^{3} \\
-u z_{0}^{5} z_{1}^{8} z_{2}^{3} z_{3}\end{array}$ & $\mathbb{P}(1,1,3,11) / \mu_{33}$ & $\frac{z_{0}^{4} z_{1}^{7} z_{2}^{2} \Omega}{F}$ & $\frac{z_{2}^{3}}{z_{0}^{6} z_{1}^{3}}$ \\
\hline $3,7,8,18$ & $\begin{array}{r}z_{0}^{12}+z_{1}^{36}+z_{2}^{9}+z_{3}^{2} \\
-u z_{0} z_{1}^{7} z_{2}^{2} z_{3}\end{array}$ & $\mathbb{P}(3,1,4,18) / \mu_{6}$ & $\frac{z_{1}^{6} z_{2} \Omega}{F}$ & $\frac{z_{2}^{2}}{z_{0}^{2} z_{1}^{2}}$ \\
\hline $5,8,9,22$ & $\begin{array}{r}z_{0}^{44}+z_{1}^{11}+z_{2}^{44}+z_{3}^{2} \\
-u z_{0}^{5} z_{1}^{2} z_{2}^{9} z_{3}\end{array}$ & $\mathbb{P}(1,4,1,22) / \mu_{22}$ & $\frac{z_{0}^{4} z_{1} z_{2}^{8} \Omega}{F}$ & $\frac{z_{1}^{2}}{z_{0}^{6} z_{2}^{2}}$ \\
\hline
\end{tabular}
dimension $\kappa=1$. We now verify all these statements for the first set

Table 2: Proof of Theorem 1.17 
of weights $\left(w_{0}, w_{1}, w_{2}, w_{3}\right)=(1,5,6,8)$. We leave the verification in the other eight cases to the reader.

According to Lemma 3.10, we have

$$
\widetilde{N}=\tilde{\mathbf{e}}_{0} \mathbb{Z}+\tilde{\mathbf{e}}_{1} \mathbb{Z}+\tilde{\mathbf{e}}_{2} \mathbb{Z}+\tilde{\mathbf{e}}_{3} \mathbb{Z}=\frac{\mathbf{e}_{\mathbf{0}}}{20} \mathbb{Z}+\frac{\mathbf{e}_{1}}{4} \mathbb{Z}+\frac{\mathbf{e}_{2}}{10} \mathbb{Z}+\frac{\mathbf{e}_{3}}{5} \mathbb{Z},
$$

and

$$
\mathbf{e}=\frac{1}{20}(1,5,6,8)=\frac{\mathbf{e}_{0}}{20}+\frac{\mathbf{e}_{1}}{4}+3 \frac{\mathbf{e}_{2}}{10}+2 \frac{\mathbf{e}_{3}}{5} ;
$$

this shows that

$$
\overline{Y_{u}^{\prime}}=\left(z_{0}^{20}+z_{1}^{4}+z_{2}^{10}+z_{3}^{5}-u z_{0} z_{1} z_{2}^{3} z_{3}^{2}=0\right) \subset \mathbb{P}(1,5,2,4) / G .
$$

It is easy to see that $G=\mu_{10}$ acts on $\mathbb{P}(1,5,4,2)$ with weights $0,5,1,6$ :

$$
z_{0}, z_{1}, z_{2}, z_{3} \mapsto z_{0}, \zeta^{5} z_{1}, \zeta z_{2}, \zeta^{6} z_{3}
$$

where $\zeta$ is a primitive 10-th root of unity.

Note that

$$
\frac{\Omega}{z_{0} z_{1} z_{2} z_{3}}
$$

is a $\mathbb{T}$-invariant, hence $G$-invariant, rational differential on $\mathbb{P}(1,5,2,4)$; it follows that

$$
\omega=\operatorname{res} \overline{Y_{u}^{\prime}}\left(z_{0} z_{1} z_{2}^{3} z_{3}^{2} \frac{\Omega}{z_{0} z_{1} z_{2} z_{3} F}\right)
$$

is a $G$-invariant regular differential on $\overline{Y_{u}^{\prime}}$. This differential must be unique since, after all, the list was produced by imposing $h^{2,0}\left(\overline{Y_{u}^{\prime}}\right)=$ $h_{c}^{2,0}\left(Y_{u}^{\prime}\right)=1$, cf. Remark 1.7.

Now we claim that the curve $C=\left(z_{3}=0\right) \subset \overline{Y_{u}^{\prime}}$ is a nonsingular curve of genus 1 . This is a completely elementary calculation. Indeed, consider the lattice

$$
N_{z_{3}}=N \cap\left(\alpha_{3}=0\right)=\mathbf{e}_{0} \mathbb{Z}+\mathbf{e}_{1} \mathbb{Z}+\mathbf{e}_{2} \mathbb{Z}+\frac{1}{4}(1,1,2,0) \mathbb{Z} ;
$$

$C$ naturally sits in the simplicial plane Proj $\mathbb{C}\left[N_{z_{3}}\right] ;$ using Lemma 3.10 again, we can write

$$
C=\left(x_{0}^{4}+x_{1}^{4}+x_{2}^{2}=0\right) \subset \mathbb{P}(1,1,2) / \mu_{2}
$$

where $\mu_{2}$ acts diagonally as $\left(x_{0}, x_{1}, x_{2}\right) \mapsto\left(x_{0},-x_{1},-x_{2}\right)$. The map to the quotient is étale over $C$; this description hence shows that $C$ is a nonsingular curve of genus 1 . This proves, in particular, that the 
Kodaira dimension of $\overline{Y_{u}^{\prime}}$ is $\geq 1$; indeed, the curve $\mathrm{C}$ is contained in the zero locus $\left(z_{2}^{2} z_{3}=0\right)=Z(\omega)$ of the differential $\omega$ and, because $C$ is not a rational curve, $C$ survives to give a nontrivial element in the canonical class of the minimal model of $\overline{Y_{u}^{\prime}}$.

To conclude, it remains to show that the map

$$
m=\frac{z_{3}^{2}}{z_{0}^{4} z_{2}^{2}}: \overline{Y_{u}^{\prime}} \rightarrow \mathbb{P}^{1}
$$

is an elliptic fibration. First of all, we observe that the map is well defined, that is, the monomial defining it is $G$-invariant or, which is the same, it is an element of $N$ of degree 0 . But indeed

$$
\frac{z_{3}^{2}}{z_{0}^{4} z_{2}^{2}}=\frac{z_{0} z_{1} z_{2}^{3} z_{3}^{2}}{z_{0}^{5} z_{1} z_{2}^{5}} \quad \text { is the vector } \quad \mathbf{e}-\frac{1}{4}(1,1,2,0) \in N
$$

of degree $1-1=0$. Thus, $m: \overline{Y_{u}^{\prime}} \rightarrow \mathbb{P}^{1}$ is a well defined map. The vectors $x_{i}=\mathbf{e}_{i}, y=\mathbf{e} \in \mathbb{C}[N \cap I]_{1}$ define a birational morphism $\pi: \mathbb{P} \rightarrow \mathbb{P}^{4}$ with image the non-normal hypersurface

$$
y^{20}-x_{0} x_{1}^{5} x_{2}^{6} x_{3}^{8}=0 .
$$

The image $\pi\left(\overline{Y_{u}^{\prime}}\right)$ is the complete intersection:

$$
\left\{\begin{array}{l}
y^{20}-x_{0} x_{1}^{5} x_{2}^{6} x_{3}^{8}=0 \\
-u y+x_{0}+x_{1}+x_{2}+x_{3}=0
\end{array} \quad \subset \quad \mathbb{P}^{4} .\right.
$$

Note that

$$
\frac{z_{3}^{2}}{z_{0}^{4} z_{2}^{2}}=\frac{z_{0} z_{1} z_{2}^{3} z_{3}^{2}}{z_{0}^{5} z_{1} z_{5}}=\frac{x_{1} x_{2} x_{3}^{2}}{y^{4}}
$$

therefore, omitting coefficients, and substituting $y^{4}=x_{1} x_{2} x_{3}^{2}$ in the first line of Equation (5), we need to check that

$$
\left\{\begin{array}{l}
x_{3}^{2}-x_{0} x_{2}=0 \\
-y+x_{0}+x_{1}+x_{2}+x_{3}=0 \quad \subset \quad \mathbb{P}^{4} \\
y^{4}-x_{1} x_{2} x_{3}^{2}=0
\end{array}\right.
$$

is birational to a curve of genus 1 . This is an elementary verification: eliminating $x_{1}$ and then $x_{0}$ we obtain

$$
y^{2}+y\left(x_{3}^{2}+x_{3} x_{4}\right)+x_{3}^{4}+x_{4}^{4}=0
$$

which is, manifestly, the equation of a curve of genus 1 . 


\section{References}

[AKO] Denis Auroux, Ludmil Katzarkov, and Dmitri Orlov. Mirror symmetry for weighted projective planes and their noncommutative deformations, arXiv.org:math.AG/0404281.

[Bat94] Victor V. Batyrev. Dual polyhedra and mirror symmetry for Calabi-Yau hypersurfaces in toric varieties. J. Algebraic Geom., 3(3):493-535, 1994.

[BGP] Jim Bryan, Tom Graber, and Rahul Pandharipande. The orbifold quantum cohomology of $\mathbb{C}^{2} / \mathbb{Z}_{3}$ and Hurwitz-Hodge integrals, arXiv.org:math.AG/0510335.

[BH89] F. Beukers and G. Heckman. Monodromy for the hypergeometric function ${ }_{n} F_{n-1}$. Invent. Math., 95(2):325-354, 1989.

[CCLT] T. Coates, A. Corti, Y.-P. Lee, and H.-H. Tseng. Small quantum orbifold cohomology of weighted projective spaces. In preparation.

[DK86] V. I. Danilov and A. G. Khovanskiı. Newton polyhedra and an algorithm for calculating Hodge-Deligne numbers. Izv. Akad. Nauk SSSR Ser. Mat., 50(5):925-945, 1986.

[Dol82] Igor Dolgachev. Weighted projective varieties. In Group actions and vector fields (Vancouver, B.C., 1981), volume 956 of Lecture Notes in Math., pages 34-71. Springer, Berlin, 1982.

[Gol01] V. V. Golyshev. Riemann-Roch variations. Izv. Ross. Akad. Nauk Ser. Mat., 65(5):3-32, 2001.

[IF00] A. R. Iano-Fletcher. Working with weighted complete intersections. In Alessio Corti and Miles Reid, editors, Explicit birational geometry of 3-folds, volume 281 of London Math. Soc. Lecture Note Ser., pages 101-173. Cambridge University Press, Cambridge, 2000.

[Ish99] Shihoko Ishii. The minimal model theorem for divisors of toric varieties. Tohoku Math. J. (2), 51(2):213-226, 1999.

[Kat90] Nicholas M. Katz. Exponential sums and differential equations, volume 124 of Annals of Mathematics Studies. Princeton University Press, Princeton, NJ, 1990.

[Kol92] János Kollár. Flips and abundance for algebraic threefolds. Société Mathématique de France, Paris, 1992. With the collaboration of D. Abramovich, V. Alexeev, A. Corti, L.-Y. 
Fong, A. Grassi, S. Keel, T. Luo, K. Matsuki, J. McKernan, G. Megyesi, D. Morrison, K. Paranjape, N.I. ShepherdBarron and V. Srinivas. Papers from the Second Summer Seminar on Algebraic Geometry held at the University of Utah, Salt Lake City, Utah, August 1991, Astérisque No. 211 (1992).

[Rei87] Miles Reid. Young person's guide to canonical singularities. In Algebraic geometry, Bowdoin, 1985 (Brunswick, Maine, 1985), volume 46 of Proc. Sympos. Pure Math., pages 345414. Amer. Math. Soc., Providence, RI, 1987.

[Rei02] Miles Reid. La correspondance de McKay. Astérisque, (276):53-72, 2002. Séminaire Bourbaki, Vol. 1999/2000. 